CARTA AL EDITOR

\section{BURNOUT Y CALIDAD DE VIDA PROFESIONAL EN OPERADORES DE JUSTICIA QUE ATIENDEN VIOLENCIA FAMILIAR}

\section{BURNOUT AND QUALITY OF PROFESSIONAL LIFE IN JUSTICE OPERATORS THAT ATTEND FAMILY VIOLENCE}

\author{
Agueda Muñoz del Carpio Toia (i) 1,a, Lucía Begazo \\ Muñoz del Carpio (i) 1,b, Brunella Díaz San Román (10) ${ }^{1, c}$, \\ Alexandra Rodríguez Flores (1) ${ }^{1, c}$ Gabriela Mendoza \\ Vicuña (1) 1,c, Germán Málaga (1) ${ }^{2, d}$ \\ 1 Universidad Católica de Santa María, Arequipa, Perú. \\ 2 Universidad Peruana Cayetano Heredia, Lima, Perú. \\ a Médica cirujana, doctora en Medicina; ${ }^{b}$ estudiante de Publicidad y \\ Multimedia; ${ }^{c}$ estudiante de Derecho; ${ }^{d}$ médico especialista en Medicina \\ Interna, magíster en Medicina
}

Sr. Editor: La violencia familiar constituye un serio problema de salud pública que afecta principalmente a mujeres de todas las edades, y sus modalidades van desde maltrato hasta el feminicidio ${ }^{(1)}$. En el Perú, se la asocia a la falta de denuncia y al silenciamiento cultural ${ }^{(2)}$; esta situación ha obligado al Estado a establecer políticas públicas y normas legales para proteger a las mujeres ${ }^{(3)}$. Ello así, se han creado instancias donde se reciben las denuncias, se investigan y resuelven casos, donde trabajan personas que escuchan historias reales de violencia y dolor, y están día a día expuestas a riesgos psicosociales que causan desgaste laboral, agotamiento emocional, despersonalización y pérdida del sentido de la profesión $^{(1)}$.

Se buscó determinar la frecuencia del síndrome de Burnout y de la percepción de calidad de vida profesional y establecer su posible asociación en operadores de justicia que atienden casos de violencia familiar en la región de Arequipa. Se realizó un estudio descriptivo transversal para evaluar la frecuencia del Burnout a través del inventario de Burnout de Maslach, versión MBIHUMAN Servives Survey (MBI-HSS), instrumento que consta de 22 preguntas con 7 opciones de respuesta tipo escala Likert (0 para ausente y 6 si ocurre todos los días), considerándose como síndrome de Burnout o desgaste laboral cuando las respuestas

Citar como: Muñoz del Carpio Toia A, Begazo Muñoz del Carpio L, Díaz San Román B, Rodríguez-Flores A, Mendoza-Vicuña G, Málaga G. Burnout y calidad de vida profesional en operadores de justicia que atienden violencia familiar. Rev Peru Med Exp Salud Publica. 2020;37(3):589-90. doi: https://doi. org/10.17843/rpmesp.2020.373.5276

Correspondencia: Agueda Muñoz del Carpio Toia, amunozde@ucsm.edu.pe

Recibido: 18/02/2020 Aprobado: 24/06/2020 En línea: 04/08/2020 alcanzaron altas puntuaciones en las dimensiones (agotamiento emocional y despersonalización) y bajas puntuaciones en la dimensión realización personal en el trabajo ${ }^{(4)}$. Se aplicó además un test de evaluación de calidad de vida profesional (CVP-35), el cual consta de 35 ítems, 12 preguntas evalúan demandas, 22 analizan recursos y una valoración global de la calidad de vida según el puesto de trabajo. Las opciones de respuestas son tipo escala de Likert del 1 a 10, con 4 subcategorías ascendentes según el valor obtenido que van de nada a mucho ${ }^{(5)}$.

Se encuestaron 50 operadores de justicia de Arequipa, lo cual, abarcó al 85\% de los jueces del único módulo de violencia familiar de la región, inaugurado en octubre del 2018 como proyecto piloto a nivel nacional para disminuir la carga procesal de los juzgados de familia y que atiende todos los casos de violencia familiar de Arequipa. Se aplicó estadística descriptiva para la determinar la frecuencia de las respuestas sobre cada dimensión del Burnout, y de calidad de vida profesional.

Se utilizó la prueba de correlación de Spearman para evaluar su asociación. El proyecto fue aprobado por el Comité Institucional de Ética de la investigación de la Universidad Católica de Santa María.

De los 50 operadores de justicia, $52 \%$ eran varones, $74 \%$ presentaron nivel alto e intermedio de desgaste laboral. En cuanto a su calidad de vida profesional, esta fue alta o muy alta, medida en todas sus esferas: motivación intrínseca, apoyo directivo, demanda de trabajo y satisfacción (Tabla 1), lo que sugiere que el alto porcentaje hallado, no afectó la calidad de vida profesional, por lo que el análisis de correlación no fue significativo, como tampoco lo fueron las correlaciones entre cada dimensión de Burnout con calidad de vida profesional. El único hallazgo significativo fue la correlación entre el Burnout y la percepción de calidad de vida profesional $(\mathrm{Rho}=0,566$ y $\mathrm{p}<0,001)$, donde a mayor percepción de calidad de vida profesional menor presencia de desgaste laboral. No se encontraron diferencias significativas entre el género respecto a la frecuencia de desgaste laboral y frecuencia de calidad de vida profesional.

Hallamos que tres cuartas partes de operadores de justicia, sufren de niveles intermedios/altos de desgaste laboral, que podría explicarse por la constante exposición a los altos niveles de estrés que provoca la víctima en las personas que la escuchan y a la alta carga de crueldad en las acciones registradas ${ }^{(5)} \mathrm{o}$ la excesiva carga laboral diaria. En la ciudad de Lima se realizó un estudio con magistrados, fiscales y jueces, y se identificó estrés general en el 18,5\% de ellos; estrés laboral en el 33,7\%; ansiedad en el 12,5\%; y depresión en un $15 \%{ }^{(6)}$.

Las limitaciones del estudio están relacionadas a su naturaleza transversal, los resultados corresponden al momento de recolección de la información y no poder generalizar a otras jurisdicciones del país. Asimismo, la naturaleza voluntaria del estudio podría constituir un sesgo, pues quienes 
Tabla 1. Distribución de las dimensiones de Síndrome de Burnout y calidad de vida profesional en operadores de justicia que atienden víctimas de violencia familiar en Arequipa

\begin{tabular}{|c|c|c|}
\hline $\begin{array}{l}\text { Dimensiones de síndrome de } \\
\text { Burnout }\end{array}$ & Frecuencia & $\%$ \\
\hline \multicolumn{3}{|l|}{ Agotamiento emocional } \\
\hline Bajo & 28 & 56 \\
\hline Intermedio & 14 & 28 \\
\hline Alto & 8 & 16 \\
\hline \multicolumn{3}{|l|}{ Despersonalización } \\
\hline Bajo & 26 & 52 \\
\hline Intermedio & 9 & 18 \\
\hline Alto & 15 & 30 \\
\hline \multicolumn{3}{|l|}{ Realización personal } \\
\hline Bajo & 11 & 22 \\
\hline Intermedio & 10 & 20 \\
\hline Alto & 29 & 58 \\
\hline Total & 50 & 100 \\
\hline \multicolumn{3}{|l|}{ Motivación intrínseca } \\
\hline Deficiente & 2 & 4 \\
\hline Regular & 2 & 4 \\
\hline Buena & 33 & 66 \\
\hline Excelente & 13 & 26 \\
\hline \multicolumn{3}{|l|}{ Apoyo directivo } \\
\hline Deficiente & 1 & 2 \\
\hline Regular & 11 & 22 \\
\hline Buena & 32 & 64 \\
\hline Excelente & 6 & 12 \\
\hline \multicolumn{3}{|l|}{ Demanda de trabajo } \\
\hline Deficiente & 2 & 4 \\
\hline Regular & 17 & 34 \\
\hline Buena & 29 & 58 \\
\hline Excelente & 2 & 4 \\
\hline Total & 50 & 100 \\
\hline \multicolumn{3}{|c|}{ Percepción calidad de vida profesional } \\
\hline Deficiente & 9 & 18 \\
\hline Regular & 11 & 22 \\
\hline Buena & 26 & 52 \\
\hline Excelente & 4 & 8 \\
\hline Total & 50 & 100 \\
\hline
\end{tabular}

estuvieran desmotivados pudieran haberse abstenido. Sin embargo, el presente estudio también es una primera mirada a una población de operadores de justicia, que no solo tienen que lidiar con la crudeza de lo que representa la violencia familiar, sino que, dada la naturaleza de los crímenes implicados, están siendo escrutados muy de cerca por la población en los medios de comunicación y redes sociales.

Concluimos que los operadores de justicia en violencia familiar tienen alto porcentaje de desgaste laboral y que, a mayor percepción de calidad de vida profesional, menor presencia de Burnout, lo que abogaría a favor de la implementación de políticas de mejora en clima laboral y disminución de carga procesal.

Conflictos de interés: Los autores declaran no tener conflictos de interés.

Financiamiento: El presente estudio fue financiado por el Vicerrectorado de Investigación de la Universidad Católica de Santa María.

Contribuciones de autoría: AMCT, LBMC, BDSR, ARF, GMV concibieron y diseñaron el proyecto de investigación, y recolectaron datos; AMCT analizó e interpretó datos, AMCT, LBMC, BDSR, ARF y GM redactaron, revisaron y aprobaron la versión final del artículo.

\section{REFERENCIAS BIBLIOGRÁFICAS}

1. Bott S, Guedes A, Goodwin M, Adams-Mendoza J. Violencia contra las mujeres en América Latina y el Caribe: análisis comparativo de datos poblaciones de 12 países. 2014 [citado el 11 de enero de 2020]. Disponible en: http://repositorio.minedu.gob.pe/handle/123456789/4109.

2. Muñoz del Carpio A, Begazo-Muñoz del Carpio L, Toia-Larsen Milena, Moreno Leiva G, Tejada Camacho I. Violencia laboral contra médicos del Servicio de Salud de Atención Primaria. Arequipa, Perú. Opción. 2019; 35(90):18-40.

3. Modificación del Reglamento de la Ley $\mathrm{N}^{\circ}$ 30364, Ley para prevenir, sancionar y erradicar la violencia contra las mujeres e integrantes del grupo familiar, aprobado por Decreto Supremo Nº09-2016-MIMP. [citado el 12 de enero de 2020]. Disponible en: https://cdn.www.gob. pe/uploads/document/file/297866/ds_004_2019_mimp.pdf.

4. Gil-Monte P. Validez factorial de la adaptación al español del Maslach Burnout Inventory General Survey. Salud Publica Mex. 2002;44(1):33-40.

5. Martín J, Cortés JA, Morente M, Caboblanco M, Garijo J, Rodríguez A. Características métricas del cuestionario de calidad de vida profesional (CVP-35). Gac Sanit. 2004;18(2):129-136.

6. Perales A, Chue H, Padilla A, Barahona L. Estrés, ansiedad y depresión en magistrados de Lima, Perú. Rev Perú Med Exp Salud Publica. 2011;28(4): 581-588. 